\title{
A Study of Comprehensive Geriatric Assessment In Elderly Patients in Rural Bangalore
}

\author{
Shashidhar $\mathbf{G}^{1}$, Sivaranjani $\mathrm{K}^{2}$, Nihal Kumar Reddy Ammatalli ${ }^{3}$ \\ ${ }^{1}$ Associate Professor, ${ }^{2}$ Post Graduate, ${ }^{3}$ Post Graduate, Department of General Medicine, MVJ Medical College and Research \\ Hospital, India
}

Corresponding author: Dr. Sivaranjani K, Post Graduate Final Year, Department of General Medicne, MVJ Medical College and Research Hospital, Dandupalya, Hoskote, Bangalore Rural, Karnataka, India 562114

DOI: 10.21276/ijcmsr.2018.3.3.4

How to cite this article: Shashidhar G, Sivaranjani K, Nihal Kumar Reddy Ammatalli. A study of comprehensive geriatric assessment in elderly patients in rural Bangalore. International Journal of Contemporary Medicine Surgery and Radiology. 2018;3(3):C17-C20.

\section{A B S T R A C T}

Introduction: Comprehensive geriatric assessment (CGA) is defined as a multidisciplinary diagnostic and treatment process that identifies medical, psychosocial, and functional limitations of a frail older person in order to develop a coordinated plan to maximize overall health with aging. The objective of our study was to conduct a comprehensive geriatric assessment in elderly patients admitted to MVJMC and RH and to find out geriatric syndromes.

Material and Methods: The study was an observational study on 70 elderly patients. A proforma which included patient biodata, a routine clinical examination and a comprehensive geriatric assessment was done after interviewing and examining the patients.

Results: A total of 70 elderly individuals (41males and 29 females) were included in the study. They are classified as young old (aged 60 - 70 years), old old (aged 70 - 80 years) and oldest old (aged 80 years and above). In our study $44 \%$ of the patients were young old, $39 \%$ were old old and $17 \%$ were oldest old. The mean age of the subjects was $71.2 \pm 5.4$ years (ranged $60-92$ years). It was found that $17.1 \%$ of the patients had depression. ADL score increased progressively with age. Around $14.28 \%$ had dementia. It was found that $31.42 \%$ had a high risk of falls.

Conclusion: Our study highlights the importance of CGA being an essential part of the evaluation of elderly individuals so that the unique problems of this population are identified and addressed. Our study emphasizes the need for a Comprehensive Geriatric Assessment in elderly patients.

Key words: Frailty, Dementia, Depression, Falls, Activities of Daily Living score

\section{INTRODUCTION}

Frailty is a complex interplay of health and illness, attitudes, resources and dependence on others, leading to a decreased ability to withstand illnesses without loss of function. This increases with age. The challenge of aging to medical care lies in the complexity of frailty. As people age, it is not just that any given illness becomes more common, all illnesses become more common. Age-related changes, whether they cross a disease threshold or not, follow, on average, a trajectory of decline. Managing a single illness is tricky enough, but with the complexity imposed by frailty, managing illnesses in the presence of multiple interacting medical and social problems, each becoming more common with age, requires a specialized body of knowledge and skills. ${ }^{1}$ India is in a phase of demographic transition. ${ }^{2}$ According to National Census 2011, there are nearly 104 million elderly people in India; 53 million being females and 51 million males as compared to 20 million in 1951. There has been a sharp increase in the number of elderly persons between 1991 and 2001 and it is projected that by the year 2050, the number of elderly people would rise to about 324 million. $^{2}$ With a comparatively young population, India is still poised to become home to the second largest number of older persons in the world. India has thus acquired the label of "an ageing nation" with 7.7\% of its population being above the age of $60 \mathrm{yrs}$.

In Elderly patients, hospitalization is characterised by unpredictable outcomes often different from the actual outcome of the initial diagnosis of the patients.

Falls are a major focus of geriatric medicine because they are common among older adults, have complex interacting causes and serious consequences, requiring multiple disciplines for an effective management. The ability to use sensory information from any part of the body to promote awareness of body position is termed haptic sensation using assistive devices. Urinary incontinence is also common symptom in older adults and has a profound negative impact on the quality of life.

Clinicians should also strive to conduct periodic systematic reviews of older patients'medication regimens as well as adhere to other principles to optimize geriatric pharmacotherapy. Major causes of blindness are primarily cataract, glaucoma, and retinal diseases (mostly macular degeneration and diabetic retinopathy), all of which are strongly related to 
advancing age. ${ }^{1}$

When older adults are unable to communicate because of untreated or unrecognized hearing loss (HL), the health consequences are dramatic, especially among those with multimorbidity. Ironically, $\mathrm{HL}$ is considered a little recognized consequence of aging, despite the physical, psychosocial, and cognitive correlates of this unavoidable aspect of aging.

Older adults may present symptoms of forgetfulness and apathy that relate to depression, dementia or both. In patients with depression, significant cognitive impairment is more common in older adults than in younger adults ${ }^{3,4,5}$ and the severity of cognitive impairment on neuropsychological tests falls somewhere between normal controls and those with early Alzheimer disease.

Dementia is a critical public health problem worldwide, especially as the population ages. Dementia generally has been defined in terms of multifocal cognitive impairment sufficient to impair function and typically is distinguished from cognitive decline of lesser severity and impact.

Multifactorial and multidisciplinary interventions are essential to manage impaired mobility in older adults who are at the frailer end of the health spectrum.

Comprehensive geriatric assessment (CGA) would benefit the elderly. It is a clinical approach for assisting the elderly to achieve their best possible health and the highest level of function.There are conditions such as the failure to thrive, falls, adverse drug effects or changes in mental status which are less likely to be reported and hence the discharge diagnosis might not quote the actual reasons for hospital admission nor explain the hospital course and subsequent health status of the patient. A comprehensive geriatric assessment (CGA) is a multi-dimensional inter-disciplinary diagnostic process focused on determining a frail elderly person's medical, psychological and functional capability in order to develop and co-ordinate an integrated plan for treatment and longterm follow-up. ${ }^{6}$

With advancing age, there is an age-related increase in comorbidity and disability. Ageing is a process that steadily reduces physiological reserve, resulting in diminished ability to compensate for the toll of illness. Illnesses accumulate with age and increase in severity and number.

\section{MATERIAL AND METHODS}

70 elderly individuals admitted to MVJMC and RH were taken up for the study. Patients aged $\geq 60$ years were included. Patients who were critically ill and the ones which had an acute inter-current illness were excluded. Data was collected with the aid of a proforma, which included patient bio-data, a routine clinical examination and the Comprehensive Geriatric Assessment protocol. Core components of comprehensive geriatric assessment (CGA) that should be evaluated during the assessment process are Functional capacity,Fall risk, Cognition, Mood, Polypharmacy, Social support, Financial concerns, Advanced care preferences. Additional components may also include evaluation of Nutrition/weight change, Urinary continence, Sexual function, Vision/hearing, Dentition, Living situation, Spirituality. The Geriatric Depression Scale (GDS) is a 30-item self-report assessment which was used to identify depression in the elderly. The Mini-Mental State Exam (MMSE) was used test of cognitive function

; it includes tests of orientation, attention, memory, language and visual-spatial skills. The Short Physical Performance Battery that assess gait and balance, several simple tests like timed up and go test, get up and go test and Gait speed assessment, Office based maneuvers, Functional reach test-a test of balance that can predict future falls were done to assess falls risk. The standard method of screening for visual problems using snellen chart was done. For hearing loss screening tests included audioscopic examination, single screening question, whisper test. These were filled after interviewing and examining the patients.

\section{STATISTICAL ANALYSIS}

Statistical analysis was done using EPI Info 6 software. Students $\mathrm{T}$ test was used to study the differences between means of numerical data. Chi square test was used to assess categorical variables.

\section{RESULTS}

An observational study was conducted on elderly patients admitted to MVJMC and RH, a total of 70 individuals (41 males and 29 females) were included in the study. The elderly were classified as young old (60 - 70 years), old old (70-80 years) and the oldest old (80 years and above). In our study $44 \%$ were young old, $39 \%$ were old old and $17 \%$ were oldest old. The mean age of the subjects was $71.2 \pm 5.4$ years (Range 60-92 years). Fifty eight percent of the subjects were male (mean age $69.4 \pm 7.3$ years) and $42 \%$ females (71.1 \pm 6.1 years). (Fig 1)

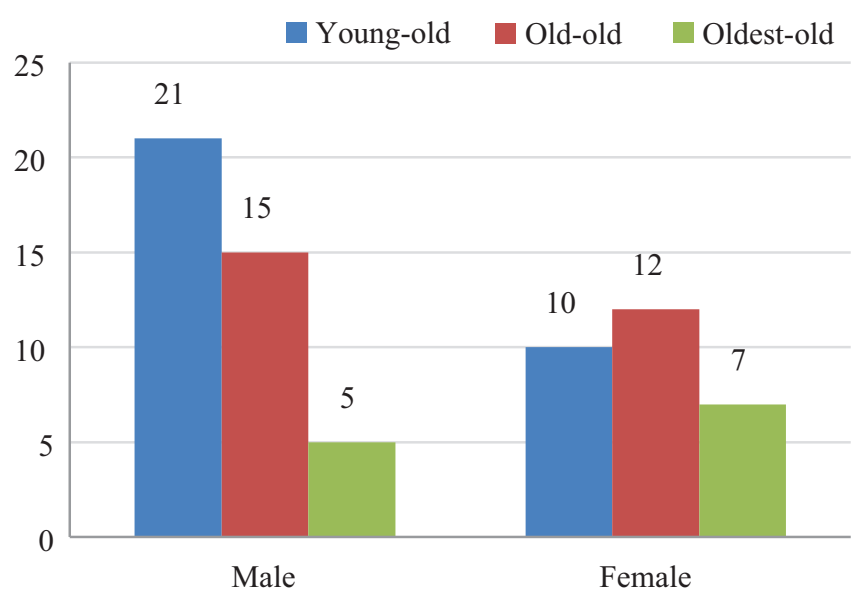

Figure-1: Gender based age distribution

\begin{tabular}{|l|c|c|}
\hline Depression & No. of patients & $\%$ \\
\hline Yes & 12 & 17.14 \\
\hline Possible & 16 & 22.85 \\
\hline No & 42 & 60 \\
\hline \multicolumn{2}{|c|}{ Table-1: Prevalence of Depression in the study group } \\
\hline
\end{tabular}

\begin{tabular}{|l|c|c|}
\hline Dementia & No. of patients & $\%$ \\
\hline Yes & 10 & 14.28 \\
\hline No & 60 & 85.71 \\
\hline \multicolumn{3}{|c|}{ Table-2: Prevalence of Dementia in study group } \\
\hline
\end{tabular}




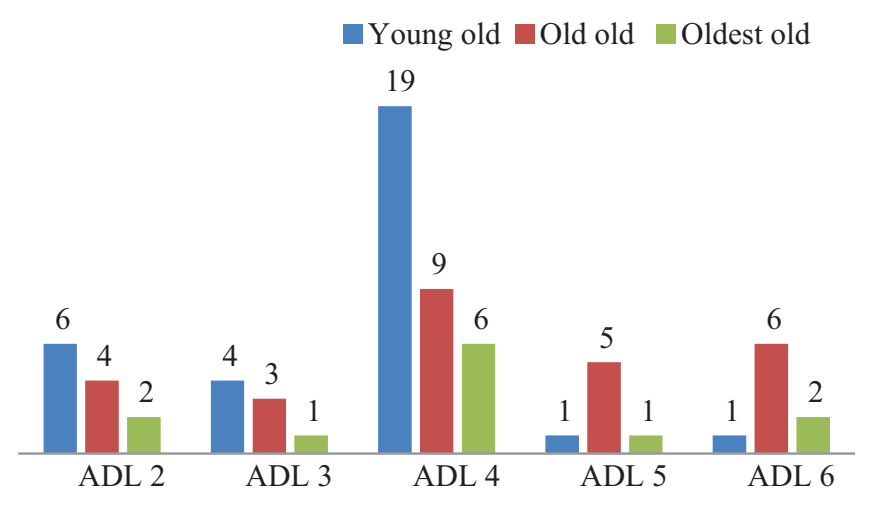

Figure-2: ADL score in different age groups

Seventy eight percent of the subjects were widowers. This finding was uniform across the three age groups. All the individuals that were studied were literate, with $11.42 \%$ of the individuals being graduates. Seventy five percent of the subjects took medications on their own. In our study, the mean number of co-morbidity found was $2.6 \pm 0.7 ; 1-3$ co-morbidities were found in $85.7 \%$ of the subjects and $10 \%$ of the subjects had more than 3 co-morbidities.

Forty one percent of the individuals had a history of consuming alcohol and $18.57 \%$ of the individuals had a history of smoking. Fifty percent of the individuals had a normal Body Mass Index, 11.42\% were obese, 25.71\% were overweight and $12.85 \%$ were underweight. Thirty one percent of the subjects were found to have visual impairment and $34 \%$ of the subjects had hearing impairment. Depression was diagnosed in $10.5 \%$ of the subjects based on the GDS 15 score. Additionally, 22.85\% of the subjects had a score suggestive of possible depression, needing a close follow up. Dementia was diagnosed in $14.28 \%$ of the subjects based on the MMSE scores. The MMSE was significantly lower in the female subjects as compared to the males; $22.9 \pm$ 9.9 vs $29.2 \pm 2.1$ respectively $(p=0.0008)$. The maximum prevalence of dementia was found among the old old persons $(22.22 \%)$ as compared to the young old (6.4\%) and the oldest old (16.66\%). and there was a greater prevalence of dementia among the female subjects $(26.08 \%$ vs $10.8 \%$ $p=0.011$.

The mean ADL score increased progressively with age, though the differences were not statistically significant. The mean ADL was significantly higher in subjects over the age of 80 years as compared to those below 80 years $(4.7 \pm 2.6$ vs $2.1 \pm 1.9 \mathrm{p}=0.031$ ). An ADL score of 4 , indicating that the individuals needed significant help with their daily living activities, was found in $48 \%$ of the subjects. An ADL score of 6, indicating a complete dependence for activities of daily living, was significantly more in women $(29.1 \%$ vs $8.3 \%, P$ $=0.016$ ). A high falls risk was diagnosed in $31.42 \%$ of the subjects according to the falls risk assessment. The mean falls risk score was highest among the oldest old; young old $6.14 \pm$ 2.95 , old old $5.86 \pm 3.45$ and oldest old $7.83 \pm 3.81$. However the differences were not statistically different. The mean falls risk score was significantly higher in subjects older than 80years as compared to those below 80 years of age (7.83 \pm 3.81 vs $4.61 \pm 3.38, P=0.023$ ).

\section{DISCUSSION}

In the descriptive study on 70 elderly patients admitted to MVJ MC and RH, a comprehensive geriatric assessment was done to find out geriatric syndromes.

\section{Demographic details}

\section{Age distribution}

The age group of the individuals studied ranged from 60-92 yrs. The mean age of the subjects was $71.2 \pm 5.4$ years. In males it was $69.4 \pm 7.3$ years and in females $71.1 \pm 6.1$ years.

\section{Gender distribution}

There was an almost equal distribution of males and females in this study. This corresponds to the proportion of males and females in the general population (males : females :: 1:0.9).

\section{Educational status of study individuals}

In our study, all the patients were literate, with a minority being graduates (11.42\%). It was also found that a higher number of male subjects (17.2\%) were graduates in comparison to female subjects (10.3\%).

\section{Administration of medicines}

It was found that many of the study subjects (75\%) took medications themselves.

However, the proportion of patients who needed help with medications increased with increasing age, though the differences were not statistically significant; $40 \%$ of the young old, $27.1 \%$ of the old old and $11.42 \%$ of the oldest old.

\section{Number of co-morbidities}

In our study, the mean number of co-morbidities were found to be $2.6 \pm 0.7 ; 1-3$ co-morbidities were found in $85.7 \%$ of the subjects and $10 \%$ of the subjects had more than 3 comorbidities.

\section{Habits/Addictions}

Sixty percent of the individuals in the study group had habits like smoking and consuming alcohol. Forty one percent of the individuals had a history of consuming alcohol and $18.5 \%$ of the individuals had a history of smoking.

\section{Body mass index and obesity}

A healthy BMI ranges between 18.5 and $24.9 \mathrm{~kg} / \mathrm{m}^{2}$. People with a BMI lower than $18.5 \mathrm{~kg} / \mathrm{m}^{2}$ are considered to be underweight and those between $25.0 \mathrm{~kg} / \mathrm{m}^{2}$ and $29.9 \mathrm{~kg} / \mathrm{m}^{2}$ as overweight. In our study group, it was found that $50 \%$ of the individuals had a normal Body Mass Index; 25.7\% were overweight, $11.47 \%$ were obese and $12.85 \%$ were underweight. Mean BMI was found to be 21.6 \pm 3.7 .

\section{Impairment of vision}

In our study, around $31 \%$ of the individuals were found to have visual impairment. The prevalence of impaired vision was not significantly different across the

three age categories; $13.2 \%$ in the young old, $10.2 \%$ in the old old and $7.6 \%$ in the oldest old. Even patients aged above 80 years $(7.5 \%)$ did not have a higher prevalence of impaired vision as compared to those below 80 years of age (17.1\%). The prevalence of impaired vision was not significantly different between the male (7.3\%) and the female subjects (6.7\%).

In a study done by Kamlesh et al in 2003, 61\% of the subjects 
had visual impairment ${ }^{7}$

\section{Hearing impairment}

It was found that a large number of individuals (34\%) had a hearing impairment. There was no difference in the prevalence of impaired hearing across the three age groups. Surprisingly, the prevalence of impaired hearing in those below 80 years of age was significantly higher $38.2 \%$ vs $29.2 \%(P=0.005)$.

In a study done by Kamlesh et al in 2003, 20\% of the subjects had hearing Impairment. ${ }^{\text {? }}$

\section{Depression}

It was found that $17.1 \%$ of the individuals had depression according to the GDS 15

score and $22.85 \%$ of the individuals had a score suggestive of possible depression and needed close follow up.(Table 1). According to this study, it was found that there was no gender preponderance to depression. Twenty nine percent (9 of the 31) among the young old, twenty five percent (7 of the 27) among the old old and 33\% (4 of the 12) among the oldest old had possible or overt depression, indicating an increasing prevalence of depression with increasing age.

Worldwide, a number of studies have estimated the prevalence of depression in non-demented elderly to be $40 \%$.A study done be Griffith et al, showed that the prevalence of depression was $13 \%$ in the elderly. $8,9,10$

\section{MMSE}

Cognitive function was assessed using the MMSE questionnaire. The MMSE in the three age groups were: 27.8 \pm 5.3 in the young old, $23.8 \pm 10.5$ in the old old and 26.8 \pm 5.3 in the oldest old. The differences were not statistically significant. The MMSE in subjects above 80 years was lower as compared to those younger than 80 years $(23.9 \pm$ 9.5 vs $27.6 \pm 5.9)$ but the difference did not reach statistical significance.

The MMSE was significantly lower in the female subjects as compared to the male

subjects; $22.9 \pm 9.9$ vs $29.2 \pm 2.1(P=0.0008)$.

\section{Dementia}

It was found in our study that $14.28 \%$ of the individuals had dementia, based on their MMSE scores.(Table 2). The maximum prevalence of dementia was found among the old old $(22.22 \%)$ as compared to the young old (6.4\%) and the oldest old (16.66\%).

In a study done by Prince $M$ at Kerala, seven methodologically robust studies were identified and the prevalence of dementia was found to range from $1.3 \%$ to $5.3 \%$ for all those aged 60 or above and from $1.7 \%$ to $5.2 \%$ for all those aged 65 and above. $^{11}$

\section{Activities of Daily living}

The mean ADL score increased progressively with age, though the differences are not statistically significant; $2.1 \pm 3.2$ in the young old, $3.3 \pm 3.4$ in the old old and $4.7 \pm 2.1$ in the oldest old.(Fig 2)

\section{Risk of falls}

It was found that $31.42 \%$ of the subjects had a higher risk of falls (score of 10 or more) according to the falls risk assessment. It was found that the risk of falls existed across all the ages. The mean falls risk score was highest among the oldest old; in the young old being $6.14 \pm 2.95$, in the old old $5.86 \pm 3.45$ and in the oldest old $7.83 \pm 3.8$.

According to a study done by Kamlesh et al in 2003, 51.5\% of elderly people over 60 years old had a higher risk of falls. ${ }^{7}$

\section{Limitation of the study}

Due to the small sample size in our study, a statistically significant comparison among the three age group distributions could not be done. A larger sample size of study population would help draw a definitive pattern of presentation in the elderly population as a whole.

\section{CONCLUSION}

Our study finds out geriatric syndromes faced by the elderly and which can be captured by a Comprehensive Geriatric Assessment. The effect of the CGA should be formally demonstrated to be applied clinically and included in the treatment plan. These results can serve as a beginning for using the CGA based tools in monitoring hospitalised patients and guiding physicians in decision making and thereby improving the quality of life of the patients.

\section{REFERENCES}

1. Brocklehurst's Textbook of Geriatric Medicine and Gerontology; eighth edition; pg. 381,799,811.

2. Age care statistics.(online).2007.(cited on 2007 oct 6); available from : URL : http://www.helpageindia.com.

3. Butters MA, Whyte EM, Nebes RD, et al: The nature and determinants of neuropsychological functioning in late-life depression. Arch Gen Psychiatry 2004; 61(2):587-595.

4. Weisenbach SL, Boore LA, Kales HC: Depression and cognitive impairment in older adults. Curr Psychiatry Rep 2012; 14(4):280-288.

5. Rapp MA, Dahlman K, Sano M, et al: Neuropsychological differences between late-onset and recurrent geriatric major depression. Am J Psychiatry 2005; 162(3):691-698.

6. Devons CA. Comprehensive geriatric assessment: making the most of the aging years. Curr Opin Clin Nutr Metab Care 2002; 5(6):19.

7. Kamlesh Joshi, Rajesh Kumar and Ajit Avasthi. Morbidity profile and its relationship with disability and psychological distress among elderly people in Northern India.Int.J. Epidemiol. 2003; 32 (6): 978-987.

8. Mann AH, Graham N, Ashby D. Psychiatric illness in residential homes for the elderly: a survey in one London borough. Age Ageing. 1984;13(5):257-265.

9. Mann AH, Schneider J, Mozley CG et al. Depression and the response of residential homes to physical health needs. Int J Geriatric Psychiatry2000;15(1):1105-1012.

10. Griffiths. R.A, Good W. R, Watson N.P, Donell H. F, Fell P.J, et al.Depression, dementia and disability in the elderly. The British Journal of Psychiatry.1987;150(3): 482-493.

11. PrinceM.Methodological issues for population-based research into dementia in developing countries. A position paper from the 10/66 Dementia Research roup. Int J Geriatr Psychiatry. 2000;15(1):21-30.

Source of Support: Nil; Conflict of Interest: None

Submitted: 01-06-2018; Accepted: 24-07-2018; Published online: 28-07-2018 